\title{
Study of Error Propagation in Mesh Caused by Surface Point Movement in FVM Systems
}

\author{
Yu Cao ${ }^{1, a}$, Xiao-Wei Guo ${ }^{1, b}$, Qian Wang ${ }^{1, c}$, Miao Wang ${ }^{1, d}$, Zhiyuan Wang $^{1, \mathrm{e}}$ \\ ${ }^{1}$ State Key Laboratory of High Performance Computing, National University of Defense Technology, \\ Changsha, Hunan, China \\ aemail: caoyu@nudt.edu.cn, ${ }^{\text {bemail:guoxiaowei@nudt.edu.cn, }{ }^{c} e m a i l: w a n g q i a n @ n u d t . e d u . c n, ~}$ \\ demail:mercury.miao@nudt.edu.cn, eemail:wzy@nudt.edu.cn
}

\section{Keywords: Free surface; Discretization error; FVM; Error propagation}

\begin{abstract}
The correctness of the surface point position and the smoothness of the interface would significantly influence the final results in numerical simulations, especially in free surface cases such as bubble in the water, vessel with blood. However, how would the error caused by improper surface position and rough shape affect the result remains unknown. In fact, moving the surface point incorrectly would lead to a discretization error which depends on discretization schemes and difference methods. In this paper, the effects of the error caused by wrong displacement of one point are investigated in a system based on finite volume method (FVM). The influence and the error propagation characters of three basic arithmetic operators (first time derivative, the convection term, the Laplacian term) are studied in pipe flow like cases. The results shows different propagation properties which could guide further study of error propagation in free surface problems in the future.
\end{abstract}

\section{Introduction}

The control of the error is of great importance to guarantee the correctness of numerical simulations. There are a variety of errors needed to be considered in numerical simulations including truncation error, round-off error, dicretization error etc. Among them, the discretization error is generally the largest one. The analysis of discretization error is even more vital in free surface simulations $[1,2]$ such as bubble in the water, vessel with blood etc. Because cell size and cell shape is always changing which would lead to a varied discretization error. The accuracy of the final result is closely related to the quality of the mesh near the surface. The quality could be deteriorated either by wrong grid position caused by bad free surface adjusting algorithm or by rough interface caused by improper cell shape and size.

Besides the discretization error caused by improper surface cell shape and size, the propagation characters of the error is also important. Sometimes an error in one cell would spread quickly and lead to the simulation failure finally (e.g. rough surface in extrusion simulations), while sometimes relative large errors in unimportant areas is also acceptable (e.g. coarse mesh near the inlet in flow past cylinder simulations). Thus, investigating the propagation of the error is significant to control the error and guarantee the success of the simulation.

Researchers have proposed many indicators [3-5] to measure the mesh quality such as the aspect ratio (the ratio of cell length to cell width), skew, distortion. However, how would a low quality mesh or wrong surface would affect the simulation result and how would the loose of precision or error propagate in the mesh is still unknown according to these indicators. Two general methods are proposed to investigate the characters of error propagation. One is to investigate the characters of the matrix [6] it formed in the linearized equation. it could tell the developers whether or not the solution is trustable, however, it is hard to describe how the error is propagated in the mesh. The other one is to build up models for the propagation of the error [7]. Error propagation model directly depends on discretization schemes and difference methods [8]. For example, general discretization schemes used in continuous medium model like FEM, FVM often spread the error to all cells after one iteration. However, LBM [9], a new discrete medium model, would only pollute cells nearby. In 
terms of difference method, it determines the data dependence relation and the range that the error would pollute. For instance, two order central difference would affect its neighbour cell while one order upwind difference would only influence itself.

Though the error propagation model method could display the procedure of error propagation in a clear way, it is usually hard to build up proper models for real physical problems in practical, especially for continuous medium models. Most previous literature mainly focuses on the degree of accuracy, which is not easy to describe the influence intuitively. Based on error propagation model method, this paper investigates the propagation of error in mesh caused by surface point movement in FVM systems for pipe flow like case. The innovations of this paper are as follows:

* Analyze the factors when building up error propagation models

* Design the error source so as to investigate the influence of the error, and build up error propagation models for three commonly used arithmetic operators (first time derivative, the convection term, the Laplacian term).

* Validate the correctness of the proposed model. The results show that the propagation of the error is closely relies on discretization schemes and difference methods, sometimes it would be related to the parameters of the mesh (e.g. the aspect ratio). In addition, only spacing discretization terms (i.e. the convection term and the Laplacian term) would spread the error to other cells.

The rest of this paper is organized as follows. An overview of error model and the analysis of factors of error propagation are given in Section 2. Detailed description of error propagation model for each arithmetic operators are presented in Section 3. Experiments design and the numerical results are reported in Section 4. Previous works about error propagation study are briefly introduced in Section 5. Conclusions are drawn in Section 6.

\section{Overview}

Error model. General speaking, when tackling with a physical problem, the continues governing equations should be firstly discretized by cells. Linear equations are built up after discretization, and then the solution could be obtained by solving these discretized equations. As a matter of fact, Both the improper surface shape and the wrong grid position would lead to an error when implementing the equation discretization, thereby changing the element in the matrix when solving the linear equations and subsequently altering the final results. The organization of the matrixes of linear equations are directly depend on the discretization schemes (e.g. FVM, FEM, FDM) and the difference methods (e.g. central difference, upwind difference). Different discretization schemes and difference methods would result in diverse matrix structure, thereby presenting various error propagation characters.

Factors of error propagation. In this paper, the loose of precision because of mesh deformation and wrong position caused by improper surface adjusting algorithm on the surface are regarded as the same which is an error source on the surface. Therefore, manually movement of surface grids is adopted as the initial error source to investigate the interface shape influence and error propagation characters.

General models built up for physical problems are continues models. In order to describe the law of objective motion, a great number of continues equations are proposed. However, computers could only address discrete issues. Thus all the physical quantities should be scattered into discrete mesh at first. One of the most famous equations which is widely used in liquid simulations is the momentum equation which could be written as

$$
\rho\left(\frac{\partial u}{\partial t}+u \cdot \nabla u\right)-\beta \Delta u=c
$$

Where $\rho$ is the dense of the liquid, $u$ is the velocity, $t$ is the time, $\beta$ is the viscosity and $c$ is a constant force. Four terms are time derivative term, convection term, Laplacian term and constant term. Without loss of generality, the constant $c$ is considered as a positive value in this paper. It is a continuous equation which can only be solved after discretization. Physical quantities are usually stored on grids or the center of the cell, while values on the cell face are needed in the 
calculation. Therefore interpolation is adopted to obtain these essential variables on the cell face. Note errors are also brought in during the interpolation procedure. On the one hand, general interpolation schemes give tacit consent to the view that the value of points in the cell satisfy a certain rule, such as linear variation. However, it is often not true in the real world. Such approximation could only be considered reasonable when the distance of grids is enough short. In other words, the truncation error caused by cell size could be limited under a specific threshold by increasing the dense of the mesh. On the other hand, irregular cell figure would also lead to large discretization error. Because the gradient of the quantity on the grid or cell center are usually the arithmetic average, irregular cell figure would result in a longer distance between the interpolation points and the original grids for some points which brings in a relative larger truncation error. Such errors can only be reduced by transforming these irregular cells into regular ones. The best cell figures are considered as regular polyhedron (e.g. regular tetrahedron, regular hexahedron).

There are a variety of discretization schemes at present including FEM, FDM, FVM etc. Among them, FVM is widely used in liquid simulation because of its property of local conservation. Every term in the equation needs to do an integral by the cell volume $V$ in finite volume method, and according to Gauss's theorem, Most spatial derivative terms could be converted to integrals over the cell surface $\mathrm{S}$ bounding the volume.

$$
\int_{V} \nabla * \phi d V=\int_{S} d S * \phi
$$

Where $S$ is the surface area vector, $\phi$ could stand for any tensor field and the star notation * could represent any tensor product (i.e. inner, out and cross). It can be seen clearly from Eq. 2 that when the surface of the cell varies, both the size and the direction of the surface vector would change, and thereby influence the solution.

Besides discretizaiton schemes, equation discretization is closely related to difference methods. Eq. 3 and Eq. 4 are central difference (CD) and upwind difference (UD) respectively which are frequently-used in numerical simulations.

$$
\begin{aligned}
& \phi_{f}=f_{x} \phi_{P}+\left(1-f_{x}\right) \phi_{N} \\
& \phi_{f}= \begin{cases}\phi_{P} & \text { for } F \geq 0 \\
\phi_{N} & \text { for } F<0\end{cases}
\end{aligned}
$$

Where $\phi_{f}$ represents the physical quantity on the face center. $P$ and $N$ represent the owner cell and the neighbour cell respectively. $f_{x} \equiv \overline{f N} / \overline{P N}$ where $\overline{f N}$ is the distance between face center $f$ and cell center $N$, and $\overline{P N}$ is the distance between cell centers $P$ and $N$.

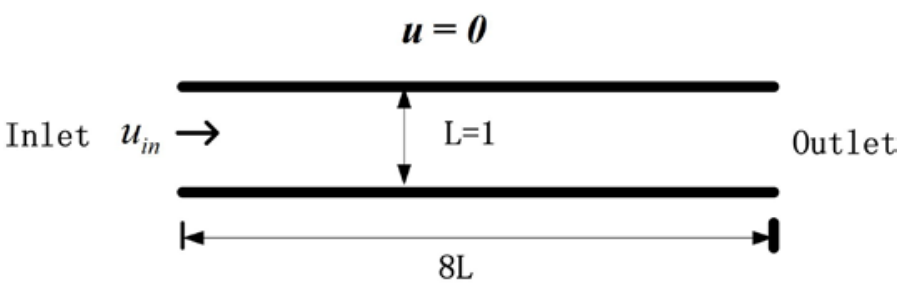

Fig. 1. Pipe flow configurations

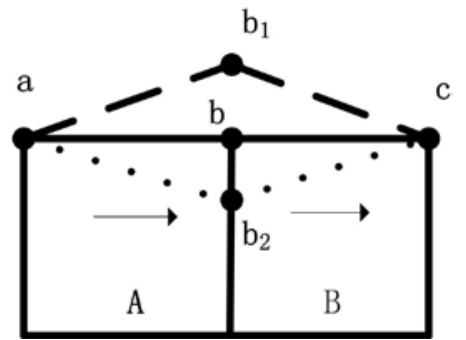

Fig. 2. One point at the surface is displaced

It can be seen from the two formulas above that physical quantities would be affected by two adjacent cells with two order central difference, while would only be affected by one cell (the upwind cell) with one order upwind difference. Note because it is hard to realize high order difference method in FVM, only order and two order difference methods are discussed in this paper. As a matter of fact, difference former determined the range of the error propagated.

This paper investigated the error propagation model of three basic term, the time derivative term, the convection term and the Laplacian term, with various difference methods based on FVM. Errors are introduced by manually moving the grids on the surface. For the simplicity of investigation, flow's behaviour in a straight pipe (see Fig. 1) is studied and only one point on the surface is misplaced along vertical direction (see Fig. 2). 


\section{Error propagation model}

Model for time derivative term. To build up models for the first time derivative, the equation $d u / d t=c$ is adopted. Integrating over a control volume for each term and using Euler implicit scheme for the first time derivative term, the form of this equation can be written as

$$
\frac{\partial}{\partial t} \int_{V} u d V=\frac{\left(u_{P} V\right)^{n}-\left(u_{P} V\right)^{o}}{\Delta t}=\int_{V} c d V=(c V)^{n}
$$

Where $\mathrm{n}$ and o represent the time step it is solving for and the previous time step respectively. The velocity of current time step can be then solved as

$$
u_{P}^{n}=c^{n} \Delta t+\left(u_{P} V\right)^{o} / V^{n}
$$

The conclusion can be drawn from Eq. 6 that if the cell volume is increased, the velocity of the corresponding cell would decrease relatively and vice versa (Note previous velocity $u_{P}^{o}$ is considered as non-zero here). Obviously, the volume of the cell would be increased when one of the grid is moved upward. What is more, the velocity of cells with the same cell volumes would increase linearly. Specifically, When c equals to zero, the velocity would keep constant. That is to say, the error would not propagation along meshes due to the first time derivative term. Therefore, the error propagation model for the first time derivative term could be depicted as the following

Model 1 The first time derivative term would not propagate the error spatially, and only cells connected to the moved grid would vary. For Euler difference method, moving the grid outwards would result in a decrease of velocity of cells connected to the moved point and vice versa.

Model for the convection term. The convection term is generally used to describe the transportation of mass, momentum or power caused by fluid convection. A typical equation can be written as $\nabla \cdot(U \alpha)=c$, Where $\alpha$ could be considered as the concentration. Integrating over a control volume, this convection term could be linearised as

$$
\frac{\partial}{\partial t} \int_{V} \nabla \cdot(u \alpha) d V=\int_{S} d S \cdot(u \alpha)=\sum_{f} S_{f} \cdot(u)_{f} \alpha_{f}=\sum_{f} F \alpha_{f}=\int_{V} c d V=c V
$$

Where $f$ present quantities on the face center and $F$ is the flux through the cell face.

Supposing the flow is uniform flow and is parallel to the cell (see Fig. 2), and the velocity of the inflow keep constant. When the grid (pint $b$ ) is moved upwards (to point $b_{1}$ ), for the cell on the left (cell A), only the right face and the upper face have changed. The flux of the right face is increased due to the increase of the area, while the flux of the upper face does not change because its boundary condition is keeping the velocity zero. Therefore, the total flux is increased. In order to maintain the sum of the product (see Eq. 7) as a const, a decrease of the concentration is required. The concentration of the cell on the right (cell B) would increase through similar analysis. Concerning with the cells remain, besides the boundary layer (cells possessing the boundary patch) with displaced grid point, all the other cells would be unaffected as there is no change of their flux. For the boundary cells after cell $\mathrm{B}$, because the contribution of the left face is negative and the magnitude of it has been increased, the concentration of their own cell should be increased accordingly. While for the boundary cells before cell A, things become interesting. If central difference method is used as Eq. 3, these cells would vary between increased and decreased alternately. This phenomena is due to the flux of the face is affected by two adjacent cells and the velocity at the inlet would never change. Therefore, cells located in the front pipe should vary in this way so as to satisfy the convection equation. While when upwind difference method is used as Eq. 4, face values would only be affected by their upwind cells, which means face flux of cells before cell A does not change in the simulation, thus the concentration is also invariant. Similar analysis could be done for the condition that the surface grid is moved downwards. To summarize the preceding analysis, error propagation model for the convection term could be given as

Model 2 The convection term would propagate the error spatially. However, in uniform pipe flow with a constant inlet velocity, only cells in the varied boundary layer may changed. Moving the grid outwards would result in a decrease of concentration of its left cell and an increase of its right 
cell. The concentration of downstream boundary layer cells would also increase while the change rule of the upstream boundary layer cells is related to the difference method.

Model for the Laplacian term. Different from the convection term, the Laplacian term is used to describe the transportation of mass, momentum or power due to random motion of molecules. Laplace operator is widely used in constructing Poisson equation which can be written as $-\nabla \cdot(\nabla u)=c$. In particular, when the constant c equals to zero, the equation is called Laplacian equation. The final discrete form of the Laplacian term is given by

$$
\frac{\partial}{\partial t} \int_{V} \nabla \cdot(\nabla u) d V=\int_{S} d S \cdot(\nabla u)=\sum_{f} S_{f} \cdot(\nabla u)_{f}
$$

If the length vector $\mathrm{d}$ between the owner cell center $\mathrm{P}$ and the neighbouring cell center $\mathrm{N}$ is orthogonal to the face plane, i.e. parallel to $S_{f}$, the face gradient discretization can be calculated as

$$
S_{f} \cdot(\nabla u)_{f}=\left|S_{f}\right| \frac{u_{N}-u_{P}}{|d|}
$$

Supposing the steady state of the flow in the pipe is a parabolic curve. Then each cell satisfies

$$
-\frac{u_{\text {upN }}-u_{P}}{d_{\text {upNP }}} s_{u p}-\frac{u_{\text {downN }}-u_{P}}{d_{\text {downNP }}} s_{\text {down }}=c V
$$

Where up and down represent the value of upper cell or face and lower cell or face respectively. When the surface gird is moved with a short distance, the velocity variety along $\mathrm{x}$ direction (the flow direction) is small which can be neglected. Therefore, Eq. 10 is still considered as the controlling equation. Supposing the mesh is uniform mesh, and if the point is then moved outwards slightly, with the approximation for cells connected to the moved point as $d_{\text {downNP }}=2 d_{\text {upNP }}$, $S_{\text {up }}=S_{\text {down }}$ and $V=2 d_{\text {upNP }} S_{\text {up }}=d_{\text {downNP }} S_{\text {down }}$, from Eq. 10, it can be obtained that the velocity of cells connected to the moved point is $u_{P}=\left(2 c d_{u p N P}{ }^{2}+u_{d o w n N}+2 u_{u p N}\right) / 3$. Moving the point outwards slightly would increase $d_{u p N P}$ (See Appendix) which would result in an increase of current cell velocity $u_{P}$.

However, if the point (point B) is moved outwards with a long distance, the area of the upper face would far larger than other faces. Then Eq. 10 of cell A can be considered as

$$
-\frac{u_{f}-u_{P}^{n}}{d_{u p N P}^{n}} s_{u p}^{n}=c V^{n}
$$

Where $u_{f}$ is the velocity of the wall boundary patch which always equals to zero. The original state of cell A is

$$
-\frac{u_{f}-u_{P}^{0}}{d_{\text {upNP }}^{o}} s_{\text {up }}^{o}-\frac{u_{\text {downN }}^{o}-u_{P}^{0}}{d_{\text {downNP }}^{o}} s_{\text {down }}^{o}=c V^{o}
$$

Supposing the length and the width of the cell are $L$ and $h$ respectively. Then for 2D model, it has $c V^{o}=L^{*} h, S_{u p}^{o}=L, d_{u p N P}^{o}=h / 2$. Because $u_{\text {downN }}^{o}>u_{P}^{o}$, from Eq. 12, it has $u_{P}^{o}>2 c h^{2}$. For a long distance movement $\Delta y$, the volume $V^{n}$ and the upper cell face area $S_{u p}^{n}$ can be approximated as $1 / 2 \Delta y L$ and $\Delta y$ respectively. Then the current velocity can be solved as $u_{P}^{n}=1 / 2 c d_{u p N P}^{n} L$ according to Eq. 11. If $1 / 2 c d_{u p N P}^{n} L<2 c h^{2}$, then it has $u_{P}^{o}>u_{P}^{n}$, i.e. the velocity is decreased for cell $\mathrm{A}$ and $\mathrm{B}$. If $u_{P}^{o}<u_{P}^{n}$ is required, it at least requires $1 / 2 c d_{u p N P}^{n} L>2 c h^{2}$. Because $d_{u p N P}^{n}>\min (h / 2, L / 2) \quad$ (see Appendix), for a conservative estimation, it requires $1 / 2 c(h / 2) L>2 c h^{2}$ and $1 / 2 c(L / 2) L>2 c h^{2}$. Then it has $L>8 h$.

In laminar flow, the flow is actually matching $-\left(\partial^{2} u / \partial y^{2}\right)=c$. Supposing the boundary 
condition is $u(0)=0$ and $u(1)=0$, then the theory resolution of this equation is $u=-\frac{1}{2} c y^{2}+\frac{1}{2} c y$. Moving the point outwards is equivalent to changing the boundary condition as $u(b)=0$, where $b>1$. Therefore, for other cells with the same position $y_{0}$, it has $u\left(y_{0}\right)=-\frac{1}{2} c y_{0}^{2}+\frac{1}{2} c b y_{0}>-\frac{1}{2} c y_{0}^{2}+\frac{1}{2} c y_{0}$. i.e. Besides the cell connected to the moved point, all other cells velocity would always increase when the point is moved outwards.

Through similar analysis, the conclusion could be drawn that when the point is moved inwards, the velocity would decrease. However, because the distance of the movement is limited (can not access the lower point), the velocity would never increase no matter how long it is moved.

In summary, the error propagation model for Laplacian term could be described as

Model 3 The Laplacian term would propagate the error along each direction. Moving the grid downwards would result in a decrease of velocity. If the grid is moved outwards with a long distant and the aspect ratio is not large (e.g. small than 4), the velocity of cells connected to the moved points would decrease while other cells velocity would increase. Otherwise, all the cells velocity would increase.

\section{Experiment}

Methodology. In order to the investigate the influence of one cell error caused by rough mesh shape or wrong point position, manually movement of surface grids is adopted as the initial error source. For the sake of laying the foundation for future research about vessel with blood, the flow is running in a simple vessel like model, i.e. a straight pipe (see Fig. 1) with wall boundary condition at both sides. The middle point $(\mathrm{x}=4)$ at the upper wall is moved up and down along the vertical direction (see Fig. 2) so as to study its influence to all directions and get rid of the influent of other boundaries (e.g. inlet, outlet). More detailed initial conditions of the flow would be described in each subsection below. A steady state with the original mesh is obtained at time 1 as the correct solution. Then the middle point is moved and the reference data is obtained at time 10 . Without loss of generality, the constant $\mathrm{c}$ is taken as 1 in the following experiments. Without specification, the mesh size is $160 * 20$ and the displacement of the movement is 0.02 cell size. All the experiments are implemented in an open source FVM-based CFD simulation tool OpenFOAM [11].

Error propagation analysis of the first time derivative term. The uniform flow begins from 1 $\mathrm{m} / \mathrm{s}$ and would speed up gradually. If no change happened, all cells velocity would accelerate at a same rate. Once one point is moved, the velocity in the pipe is shown in Fig. 3. Here red and blue represent high velocity and low velocity respectively. It can be seen clearly that only cells connected to the moved point is different from others, and moving outwards would slow down the velocity while moving inwards would speed up the velocity which perfectly match Model 1 described in Section 3.1.

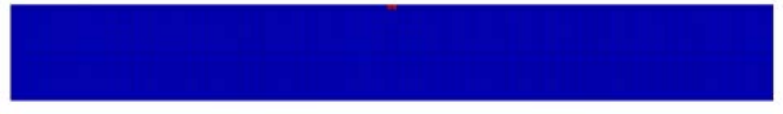

(a) The point is moved inwards

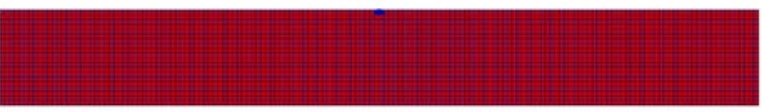

(b) The point is moved outwards

Fig. 3. Simulation results of velocity in the pipe after the middle point is moved

Error propagation analysis of the convection term. The uniform flow with $1 \mathrm{~m} / \mathrm{s}$ constant speed is used in this section to convect materials. $\alpha$ at the inlet is keeping fixed (the concentration is set as 1) as a constant source. From Model 2 depicted in Section 3.2, only cells in the varied boundary layer may have a changed concentration, and the varying pattern is depends on difference method. Therefore, the error of the concentration $\varepsilon_{\alpha}$ in the most upper cell layer with point moved inwards with central difference method, point moved outwards with central difference method and point moved outwards with upwind difference method are calculated and illustrated in Fig. 4. The simulation results show that the error of the concentration in the front area of the pipe would 
oscillate around the correct value with the same amplitude with central difference method. While there would be no error in the front area when using upwind difference method. Errors of the concentration in the back area are the same, and they are relatively small no matter how violent it fluctuated in the upstream.

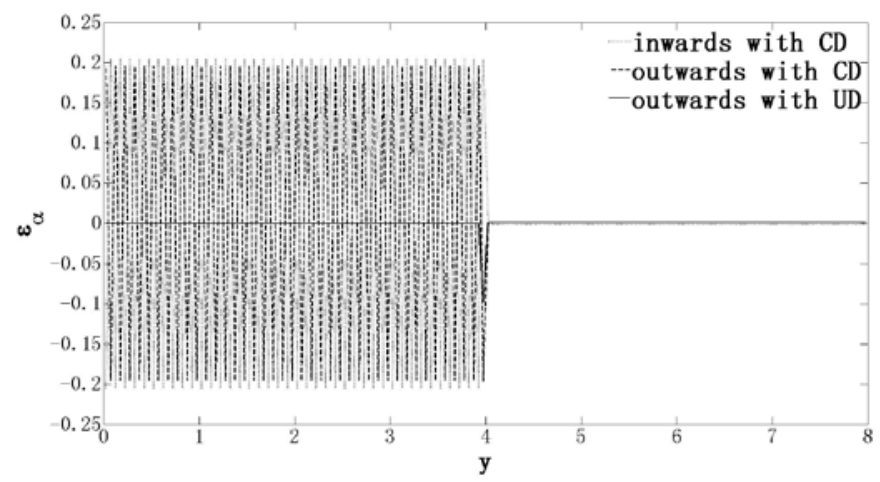

Fig. 4. Errors of the concentration in the most upper cell layer

Error propagation analysis of the Laplacian term. The flow is tend to form a parabolic curve with Laplacian term. From Model 3 demonstrated in Section 3.3, it can be known that the error would propagate to each direction. Therefore, Errors of the velocity $\varepsilon_{u}$ in the boundary layer with moved points, the layer next to the boundary layer, the vertical layer beside the moved point (two layers are the same, thus taking the left layer as an example) are extracted and shown in Fig. 5. These figures illustrate that the error would propagate along each directions indeed, and the longer distance the displacement is, the more dramatic it changes and thereby, the larger range it influences. Generally the velocity would increase as the point moved outwards and decrease as the point moved inwards as shown in Fig. 5 (b). Exceptions may occur when the point is moved outwards largely, the velocity of cells connected to the moved point may reduced as shown in Fig. 5 (a). Model 3 have demonstrates that it could be due to the mesh aspect ratio (the ratio is 1 in general cases). Simulation results with different aspect ratio are shown in Fig. 6. Here, the point is moved outwards with 5 times original cell size (i.e. moved from position $\left(\begin{array}{lll}0 & 1 & 0\end{array}\right)$ to position $\left(\begin{array}{lll}0 & 1.25 & 0\end{array}\right)$ ). Meshes size for the comparative cases are $1600 * 20$ and $16 * 20$ which means the aspect ratio is 0.1 and 10 respectively. It can be seen clearly that only when the aspect ratio is not large would a long distance outwards movement lead to a distinguishing change, which validates the correctness of Model 3.

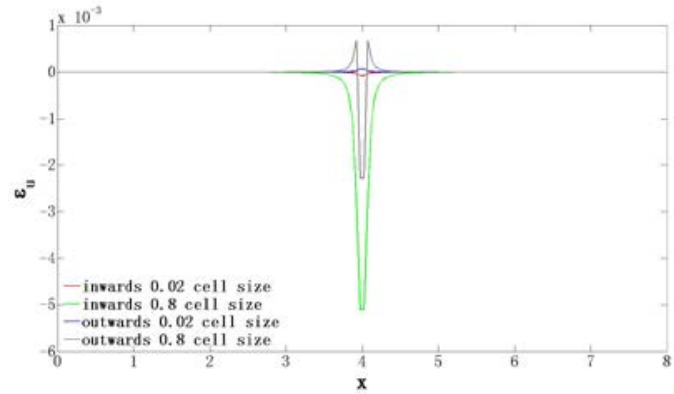

(a) The error of the boundary layer

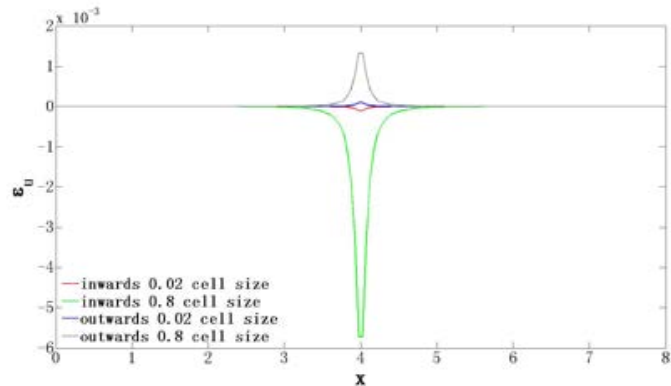

(b) The error of the layer next to the boundary layer

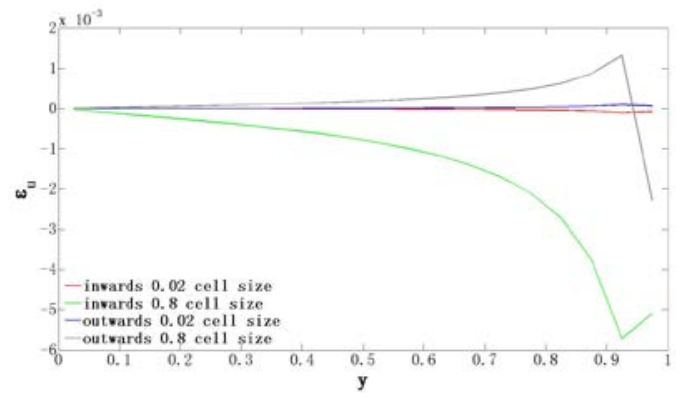

(c) The error of vertical layer beside the moved point

Fig. 5. Errors of the velocity with different direction and amplitude in various cell layers 


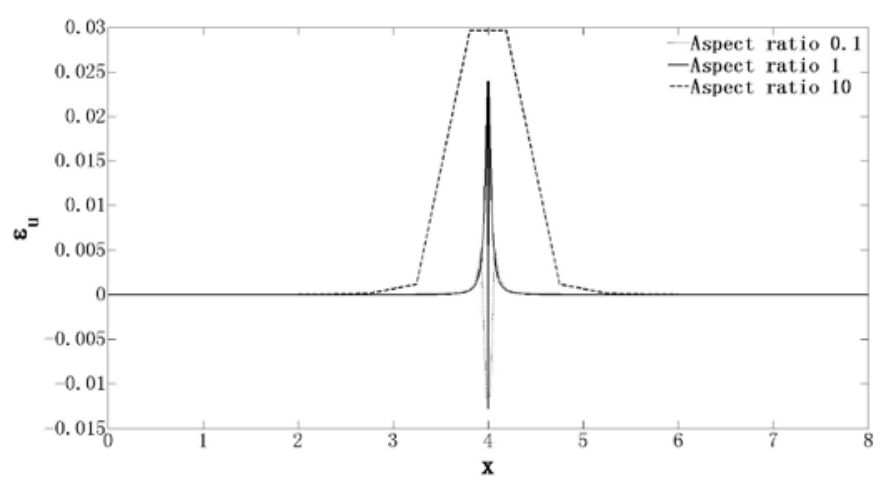

Fig. 6. Errors of the velocity with different aspect ratio

\section{Related work}

To investigate the characteristics of the error propagation, there are two frequently-used methods. One is matrix-based method [5, 6] and the other is model-based method. Matrix-based method investigate the effects of error from the perspective of numerical analysis. The influence of the error is transformed into the invetigation of the solution of the linear equation $A x=b$. The discretization error would result in an slight error in $A$ and $b$. And the deviation of the solution would heavily relay on the properties of the matrix. This method is universal and could obtain an accurate prediction of the error limit of the final solution. However, it could neither present the influence when the shape and the size of the cell have been changed, nor describe the characters of error propagation. Therefore, it could not give any suggestions to the control of error propagation. Model-based method builds up models for the propagation of errors. For example, in simulations with LBM, an error would only pollute cells around it in one time step according to the basic theory of particle collision. This character has been used for fault tolerance by Ren to design low-overhead soft error detection [7]. Therefore, model-based method could directly shed light on the law of error propagation in grids and guide the regeneration of the mesh so as to control the error. The limitation is that it might be hard to find out the potential propagation model for some cases, sometimes there might be no models for these models.

\section{Conclusion}

In this paper, the characters of error propagation in the mesh are investigated. In detail, three basic arithmetic operators (first time derivative, the convection term, the Laplacian term) are studied and different error propagation models are build up to describe them. The analysis and the experiment shows that the propagation of the error is closely relies on discretization schemes and difference methods, sometimes it would be related to the parameters of the mesh (e.g. the aspect ratio). In addition, only spacing discretization terms (i.e. the convection term and the Laplacian term) would spread the error to other cells.

However, The real physical problems are much more complex which combined a great number of arithmetic operators. What is more, the boundary condition might be more complex in some cases, and the number of displaced points may greater than one which would construct a much more complicated geometric surface shape. In the future, a real problem could be investigated so as to be more practical to guide the control of error propagation.

\section{Acknowledgement}

The authors would like to thank the funding from the National Natural Science Foundation of China under grant No.61221491, No.61303071, and No.61303068, and Open fund (No.201503-01, No.201503-02) from State Key Laboratory of High Performance Computing. 


\section{References}

[1] Tukovic, Z. and H. Jasak, A moving mesh finite volume interface tracking method for surface tension dominated interfacial fluid flow. Computers \& Fluids, 2012. 55: p. 70-84.

[2] Sommerfeld, M. and S. Decker, State of the Art and Future Trends in CFD Simulation of Stirred Vessel Hydrodynamics. Chemical Engineering \&amp; Technology, 2004. 27(3): p. 215-224.

[3] Parthasarathy, V.N., C.M. Graichen, and A.F. Hathaway, A comparison of tetrahedron quality measures. Finite Elements in Analysis and Design, 1994. 15(3): p. 255-261.

[4] Baker, T.J. Deformation and quality measures for tetrahedral meshes. in European Congress on Computational Methods in Applied Siences and Engineering, Barcelone. 2000.

[5] Gargallo-Peiró, A., et al., Defining Quality Measures for Validation and Generation of High-Order Tetrahedral Meshes. Proceedings of the 22nd International Meshing Roundtable, 2014: p. 109-126.

[6] Freitag, L.A. and P.M. Knupp. Tetrahedral element shape optimization via the Jacobian determinant and condition number. in IMR. 1999.

[7] Xiaoguang, R., et al., GS-DMR: Low-overhead soft error detection scheme for stencil-based computation. Parallel Computing, 2015. 41(0): p. 50-65.

[8] Diskin, B. and J.L. Thomas, Effects of Mesh Irregularities on Accuracy of Finite-Volume Discretization Schemes. 2012.

[9] Chen, S. and G.D. Doolen, LATTICE BOLTZMANN METHOD FOR FLUID FLOWS. Annual Review of Fluid Mechanics, 1998. 30(1): p. 329-364.

[10]Russo, G. and T.N. Phillips, Spectral element predictions of die-swell for Oldroyd-B fluids. Computers \& Fluids, 2011. 43(1): p. 107-118.

[11]Jasak, H., OpenFOAM: Open source CFD in research and industry. International Journal of Naval Architecture and Ocean Engineering, 2009. 1(2): p. 89-94.

\section{Appendix}

Supposing the surface grid is moved outwards from point $b$ to $b_{1}$ (see Fig. 2) and forming an angle $\angle b_{1} a b=\theta$. Then the new distance between the new cell center and the new face center is $\mathrm{d}_{u p N P}^{n}=\left(\frac{h}{2}+\frac{L \tan \theta}{2}\right) \cos \theta=\frac{h}{2} \cos \theta+\frac{L}{2} \sin \theta=\frac{\sqrt{h^{2}+L^{2}}}{2} \sin (\theta+\varphi)$. Where $\varphi=\arcsin \left(\mathrm{h} / \sqrt{h^{2}+L^{2}}\right)$.

Because $\theta \in\left(0^{\circ}, 90^{\circ}\right)$, it has $\min (\mathrm{h} / 2, \mathrm{~L} / 2)<\mathrm{d}_{\text {upNP }}^{n} \leq \sqrt{h^{2}+L^{2}} / 2$ 